At the Kernfysisch Versneller Instituut (KVI) in Groningen (Netherlands), H. de Waard, chairman of the Dutch FOM foundation for fundamental research on matter, signs an agreement between FOM and the French Institut National de Physique Nucléaire et de Physique des Particules (IN2P3) to build a superconducting cyclotron at Orsay for eventual installation at KVI. Looking on in the foreground is IN2P3 director $P$. Lehmann.

(Photo Nieuwsblad van het Noorden, Groningen)

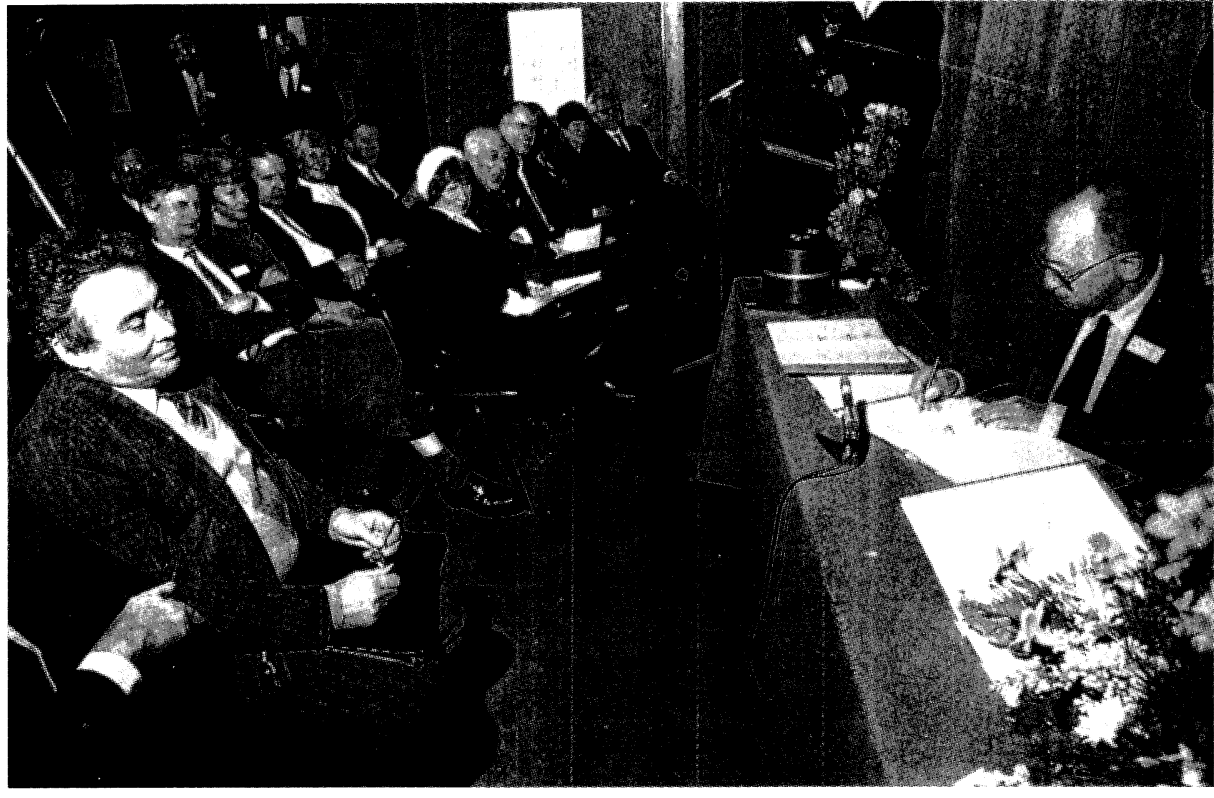

GRONINGEN/ORSAY

Superconducting collaboration

Last December a contract between the French Institut National de Physique Nucléaire et de Physique des Particules (IN2P3) and the Dutch FOM foundation for fundamental research on matter was signed in a ceremony at the Kernfysisch Versneller Instituut (KVI) in Groningen in the presence of the representative of the Dutch Minister of Science and Education and of the French Ambassador to the Netherlands.

The agreement covers a collaboration between the Institut de Physique Nucléaire (IPN) in Orsay and $\mathrm{KVI}$ in Groningen in the design and construction of a superconducting cyclotron. While the capital funds required for the new machine will be furnished by the Dutch Government, the major part of the manpower needed to build it will be supplied by the French partner. The cyclotron will be built and test- ed in Orsay and subsequently (1991) installed at KVI replacing the present $\mathrm{k}=160 \mathrm{MeV}$ cyclotron. The accelerator will be jointly exploited with approximately 20 to the French partner.

Called AGOR (Accelerateur Groningen ORsay), the new machine, based on an Orsay design, is a $\mathrm{k}$ $=600 \mathrm{MeV}$ compact variable energy fixed frequency cyclotron with superconducting coils that will be capable of accelerating both light and heavy ions: protons to $200 \mathrm{MeV}$, deuterons to $190 \mathrm{MeV}$, and totally stripped heavy ions to $95 \mathrm{MeV} / \mathrm{u}$. Moreover, it will be possible also to accelerate very heavy nuclei, and beams of polarized protons and deuterons will be available with an external source.

The scientific interest in AGOR is in the very broad range of particles it can accelerate and the wide energy range it covers. The international collaboration, at present restricted to France and the Netherlands, offers exciting new prospects. per cent of the beam time allocated

\section{TRIUMF KAON factory proposal}

The TRIUMF Laboratory at Vancouver in Canada has been operating a $500 \mathrm{MeV}$ negative ion cyclotron since 1974 mainly for nuclear physics experiments. Together with the LAMPF proton linac at Los Alamos and the SIN cyclotron in Zurich, it is one of the world's 'meson factories', producing, in particular, exceptionally intense beams of pions.

In response to today's physics challenges, the TRIUMF staff (like those at LAMPF and SIN) have been studying a major improvement of their research facilities. All three centres are looking towards much more intense beams of hadrons. The Canadian proposal is for a KAON Factory, 'KAON' being an acronym for Kaon, Antiproton, Other hadron and Neutrino.

For nuclear physics, the aim is to have a variety of beams with energies ranging from several hundred $\mathrm{MeV}$ to a few $\mathrm{GeV}$ (the ideal range for studying most nuclear phenomena). Thus the nucleus could be studied with a variety of probes - pions, nucleons, kaons and hyperons. Despite the modest energies, the bigger part of the research programme at the KAON Factory could be for particle physics as the very high intensities would allow precision measurements. Thus the KAON Factory could have much to say in neutrino physics, in particle spectroscopy and especially concerning particle symmetry principles via the study of rare decays.

The accelerator scheme aims to provide a 100 microamp proton beam $\left(6 \times 10^{14}\right.$ protons per sec- 

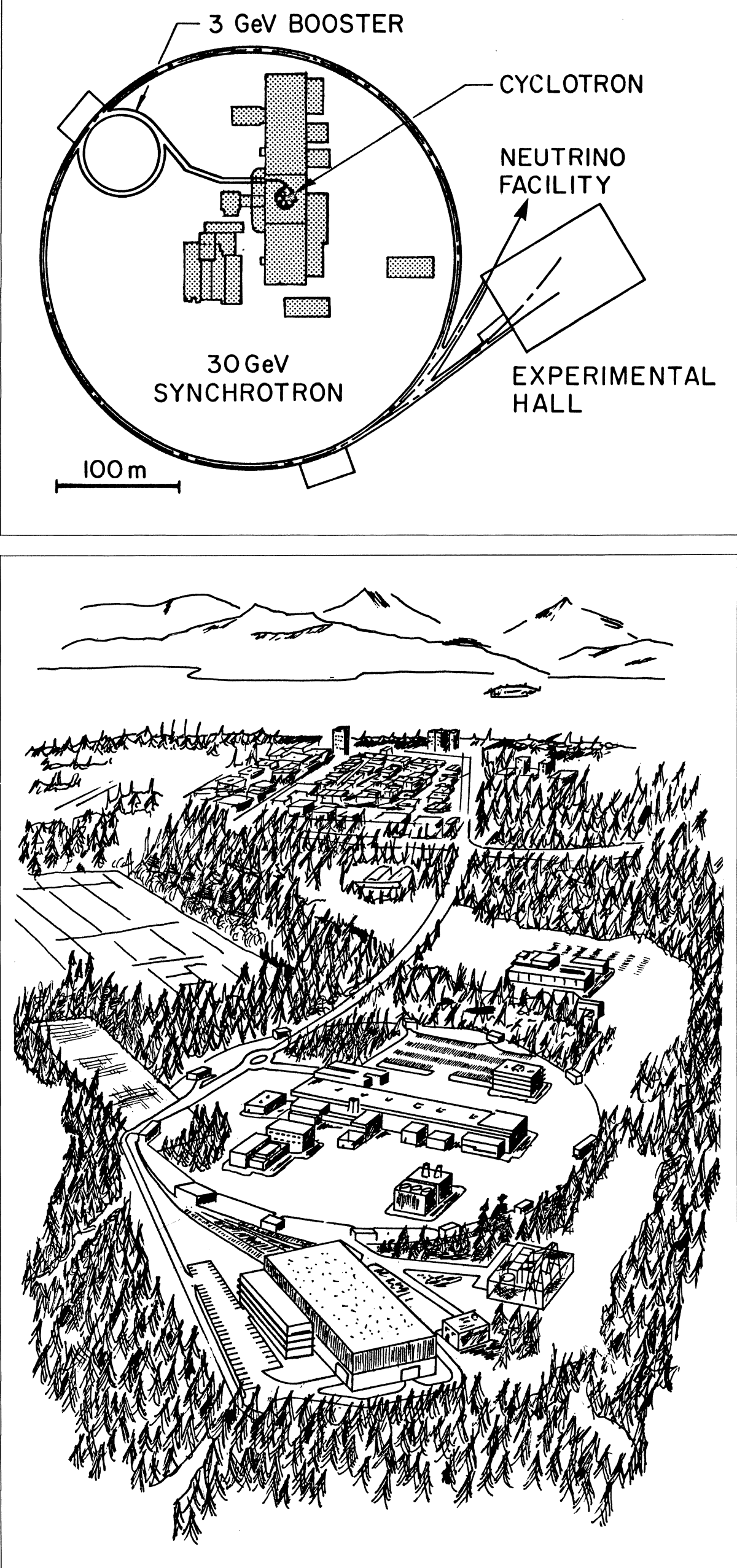

Layout of the proposed 'KAON' Factory at the Canadian TRIUMF Laboratory. The existing cyclotron would feed a $3 \mathrm{GeV}$ fast cycling booster which in turn would feed the high intensity $30 \mathrm{GeV}$ proton synchrotron built in an underground tunnel. The major visible sign of the new project would be the large experimental hall to receive the intense hadron beams.

ond) at $30 \mathrm{GeV}$; for comparison, the presently operating proton synchrotrons around that energy have intensities about fifty times lower. The scheme starts by using the existing cyclotron as a negative hydrogen ion injector into an accumulator ring where the ions are stripped to protons. Accumulation over $20 \mathrm{~ms}$ precedes transfer to a fast cycling $(50 \mathrm{~Hz})$ booster synchrotron, located in the same tunnel as the accumulator with a ring radius of $35 \mathrm{~m}$, which accelerates the particles to $3 \mathrm{GeV}$.

The protons then pass to a collector ring which receives five pulses from the booster before transferring the beam to a $30 \mathrm{GeV}$ synchrotron $(10 \mathrm{~Hz})$. Finally an extender ring receives the $30 \mathrm{GeV}$ particles for slow resonant extraction. Collector, synchrotron and extender rings are in the same underground tunnel with a ring radius of $170 \mathrm{~m}$. Obviously with such intense beams, many aspects of machine operation and component design are influenced by the need to keep beam losses down so as to have tolerable radiation levels.

A large experimental hall, $120 \mathrm{~m}$ $x 75 \mathrm{~m}$, would receive both slow and fast extracted beams. In addition there would be a conventional neutrino beam.

The cost estimates (1985 prices) are 260 million Canadian dollars for machine components and services and 80 million for construction. Experimental area facilities for some 66 million would be covered by the present level of contributions from the National

Sketch of the TRIUMF site as it would appear after construction of the proposed KAON factory. The large experimental hall is in the foreground. The undergroud synchrotron tunnel runs around the existing laboratory buildings. 
The EPOS spectrometer at the German GSI

Laboratory at Darmstadt has seen

unexplained electron-positron signals in

heavy nucleus collisions using the UNILAC

linear accelerator.

(Photo GSI)

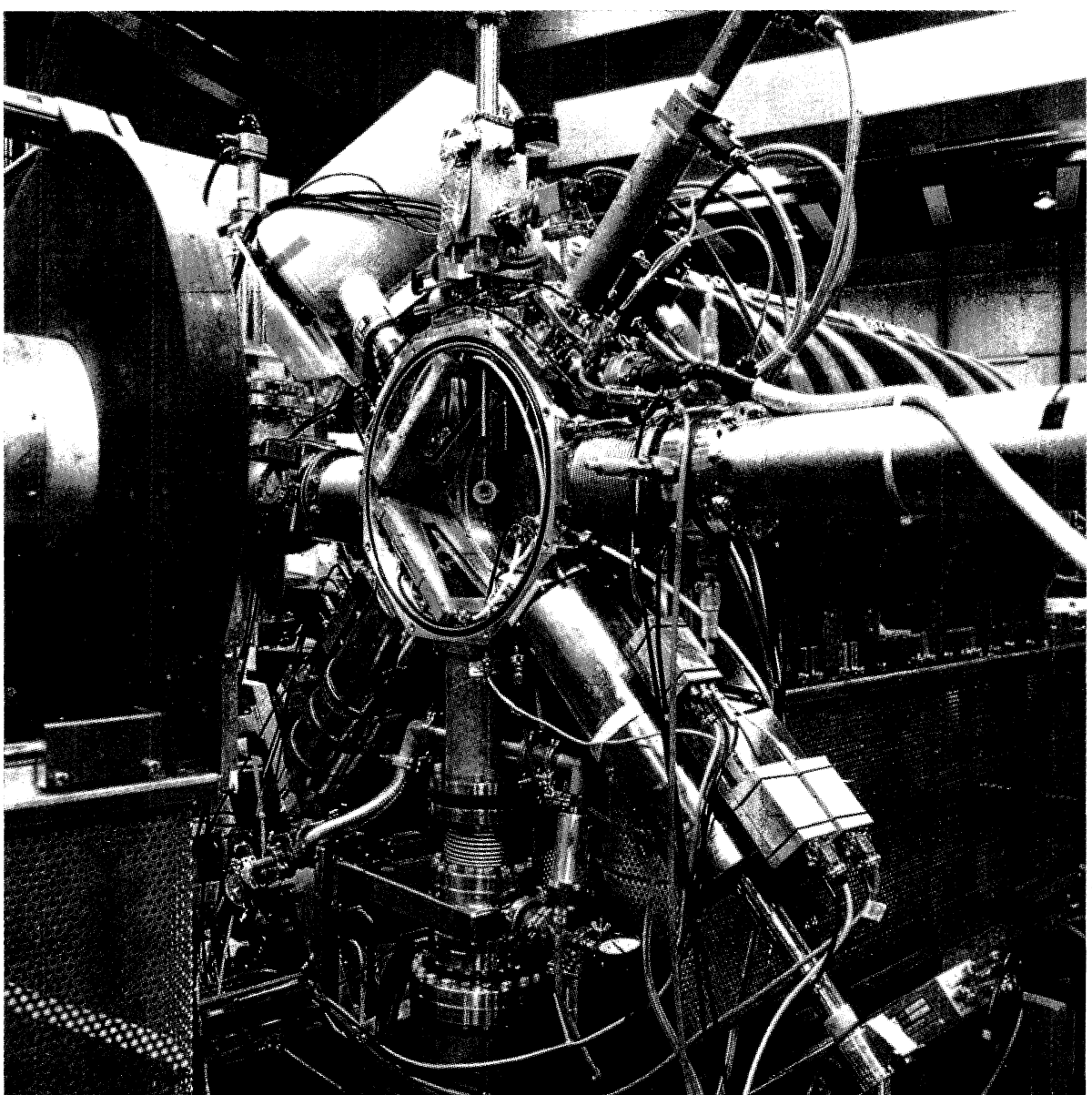

Research Council of Canada. The construction schedule is planned to cover six years for the accelerator systems plus an additional year for the experimental facilities which can be built up while the machine is being commissioned. The proposal has now been put before the Canadian Government.

\section{DARMSTADT What is it?}

For several years, experiments studying heavy nucleus collisions using the UNILAC machine at Darmstadt (GSI) have been seeing unexplained narrow peaks in the spectra of the emitted positrons. The intensity and sharpness of these peaks are too high to be accounted for by conventional nuclear processes. The position of the positron peak moreover changed little with the type of nuclear beam or target (uranium, thorium, etc). This points to these peaks having a common origin, such as a neutral particle decaying into an electron-positron pair.

To check this out, experimenters embarked on a search for electrons and positrons diverging from a common point of formation, with the EPOS spectrometer used in the positron studies modified to pick up coincident electrons.

Electron-positron coincidence events show an electron peak simi- lar to the positron signal, at an energy of $375 \mathrm{keV}$ and with a width of $75 \mathrm{keV}$.

The effect is difficult to explain through internal pair conversion of gamma rays. Detailed analysis (correlated cancellation of Doppler shifts) suggests that the particles emerge back-to-back from a common slowly moving origin in the superheavy nuclear system produced in the collision.

\section{Joint US-CERN Accelerator School}

The 2nd Joint US-CERN School on Particle Accelerators is arranged for 23-29 October in South Padre Island, Texas, with the topic 'Frontiers of Particle Beams'. The course should be of interest to both accelerator physics beginners and experienced workers. Further information from either the US Particle Accelerator School, c/o Marliyn Paul, Fermilab MS 125, PO Box 500, Batavia, Illinois 60510, USA, or Suzanne von Wartburg, CERN Accelerator School, LEP Division, 1211 Geneva 23, Switzerland.

The first Joint US-CERN Accelerator School was held last year in Sardinia on the topic of Nonlinear Dynamics (see May 1985 issue, page 140). These regular joint schools supplement the separate programmes organized by the CERN and US Schools. 